SVU- International Journal of Veterinary Sciences, 2 (2): 71-80, 2019.

Print ISSN: 2535-1826

Online ISSN: 2535-1877

CrossMark

Research Article

Open Access

\title{
Effect of Tenofovir Administration and Withdrawal on the Kidney Tissues of Adult Male Albino Rats and the Protective Role of Vitamin $\mathrm{E}$ \\ Rasha M. Abd El-Fattah ${ }^{1}$, Dorreia A. Zaghloul ${ }^{2}$, Eman A. Abd Elrahim ${ }^{3}$ and Salwa M. Ouies $^{4}$
}

${ }^{1}$ Human Anatomy and Embryology Department, Faculty of Medicine, South Valley University, Qena 83523, Egypt. ${ }^{2}$ Human Anatomy and Embryology Department, Faculty of Medicine, Assiut University, Assiut, Egypt. ${ }^{3}$ Histology Department Faculty of Medicine, South Valley University, Qena 83523, Egypt. ${ }^{4}$ Human Anatomy and Embryology, Faculty of Medicine, Sohag university, Sohag, Egypt.

\section{Abstract}

Tenofovir disoproxil fumarate is one of antiretroviral drugs used to treat chronic hepatitis B and HIV. In recent studies many Tenofovir problems were recorded; acute kidney injury (AKI), chronic kidney diseases (CKD) and Fanconi syndrome. Vitamin $\mathrm{E}$ is a powerful antioxidant that reduce free radical damage. Vitamin $\mathrm{E}$ supplementation can protect kidney tissues and enhances their regeneration if it is added to Tenofovir treatment.

The aim of the present study is to describe the changes which occur in the kidney tissues of the adult male albino rats after Tenofovir administration and determine whether these changes are reversible or not after drug withdrawal, Also, whether vitamin $\mathrm{E}$ has a protective role or not.

A total number of 40 adult male albino rats weighting 200-250 grams were used. Rats were divided into four groups, 10 rats in each group. The first group I (control group), The second group II which was treated with Tenofovir $10 \mathrm{mg} / \mathrm{kg} / \mathrm{day}$ for two months, the third group III which was treated with Tenofovir $10 \mathrm{mg} / \mathrm{kg} /$ day for two months, vitamin $\mathrm{E}$ also was given $100 \mathrm{mg} / \mathrm{kg} / \mathrm{day}$ for two months. The fourth group IV which was treated with Tenofovir $10 \mathrm{mg} / \mathrm{kg} / \mathrm{day}$ for two months then withdrawal of Tenofovir for one month.

There was marked degeneration of renal glomeruli and tubules in group II which was highly statistically significant than control group $(\mathrm{P}<0.001)$, while in group III there was marked improvement of renal glomeruli and tubules which was highly statistically significant than group II $(\mathrm{P}<0.001)$. while in group IV these changes were moderately significant when compared with group II $(\mathrm{P}<0.01)$. Therefore, Vitamin $\mathrm{E}$ has a protective role if it is added to Tenofovir treatment, also vitamin $E$ enhances regeneration of renal tissues after Tenofovir withdrawal.

Keywords: Tenofovir, Kidney, Fanconi syndrome, Vitamin E.

DOI: $10.21608 /$ svu.2019.13273.1015

Received: May 27, 2019

Accepted: June 28, 2019

Published: July 13. 2019

*Corresponding Author: Rasha A. Zaghloul

E-mail: rashanatomy@gmail.com

Citation: Abd El-Fatta et al., Effect of Tenofovir Administration and Withdrawal on the Kidney Tissues of Adult Male Albino Rats and the Protective Role of Vitamin E. SVU-IJVS 2019, 2 (2): 71-80.

Copyright: () Abd El-Fatta et al., This is an open access article distributed under the terms of the creative common attribution license, which permits unrestricted use, distribution and reproduction in any medium provided the original author and source are created.

Competing interest: The authors have declared that no competing interest exists. 


\section{Introduction}

Kidneys are highly vascularized tubular glands formed from numerous, closely packed uriniferous tubules (Bacha and Bacha, 2012). Kidneys play several critical roles in maintaining fluid and regulating electrolytes balance. They produce and activate hormones that are involved in calcium metabolism, erythrogenesis and regulation of blood pressure (Findlay and Isles, 2015). Tenofovir disoproxil fumarate (TDF) is one of the antiretroviral drugs. It has been widely used for long-term treatment of HIV and chronic hepatitis B (CHB) infections in adult patient (pham and Gallant, 2006). Tenofovir disoproxil fumarate (TDF) is an orally bioavailable pro-drug of Tenofovir (TFR), an acyclic nucleotide analogue reverse- transcriptase inhibitor (NRTI) (Buti et al., 2012). Tenofovir prevents HIV reverse transcriptase through competition with natural substrate deoxyadenosine 5'triphosphate after incorporation into DNA. (Lyseng - Williamson et al., 2005). Long term use of Tenofovir is associated with nephrotoxicity and bone loss. Presentation of nephrotoxicity can appear as Fanconi syndrome, acute kidney injury, or decline of glomerular filtration rate (GFR) (Ustianowski et al., 2015). The exact mechanism of TDF -induced nephrotoxicity is not clear but is thought to be related to the accumulation of TDF in the proximal tubules. Studies have shown that Tenofovir mainly taken up from the blood into proximal tubules cells by human organic anion transporter 1(HOAT1) at the basolateral membrane (Cihlar et al., 2001).

Vitamin $\mathrm{E}$ is a group of fat-soluble compounds with antioxidant activities, Serum concentrations of vitamin E (alphatocopherol) depend on the liver which takes up the nutrient after the various forms are absorbed from the small intestine. The liver re-secretes only alpha-tocopherol via the hepatic alpha-tocopherol transfer protein (Traber, 2006).
Vitamin $\mathrm{E}$ is a lipid soluble vitamin and therefore over $90 \%$ of total body vitamin $\mathrm{E}$ is found in the adipose tissue (Meydani et al., 1996). Vitamin E prevents reactive oxygen species which spread through biological membranes or fat when lipid content is oxidized by reacting with lipid radicals to give more stable products (Choe et al. 2009). Oxidative stress induced by the production of reactive oxygen species (ROS) during activation of polymorphonuclear leukocytes and macrophages. This is commonly occurred in HIV patients and can lead to more replication of the virus (Das et al., 1990). Supplementation of Vitamin E decreased oxidative stress and altered kidney function markers if it is added to Tenofovir treatment (Oluwatosin et al. 2012). The present study is designed to detect the effects of Tenofovir administration and withdrawal on the kidney tissues of Adult Wistar albino rats and to detect whether vitamin $\mathrm{E}$ has a protective role or not.

\section{Materials and methods}

Tenofovir was purchased from Seif Co. for chemicals and pharmaceuticals, Cairo, Egypt. Vitamin E was obtained from Pharco Company for chemicals, Cairo, Egypt.

\section{Animals and experimental design}

Forty adult male Wistar albino rats (weighing about 200-250 g and aged from 3-4 months) were obtained from the animal house, Faculty of Medicine, Assuit University and housed in a special wire cages and kept under complete isolated conditions in the animal facilities of Faculty of Medicine, Sohag University for two weeks prior to experiment for adaptation. Animals were kept on a 12-hour light, 12hour dark cycle with ambient temperature of 22-24 ${ }^{\circ} \mathrm{C}$ and were provided a commercial pelleted feed and water ad libitum. Experimental design and all animal handling procedures were approved by the Research Ethical Committee of the Faculty of Veterinary Medicine, South Valley University, Qena, Egypt (the approval no. 
20147). All precautions were taken into consideration to avoid animal suffering.

\section{Experimental design}

After acclimation, forty rats were randomly assigned to four different isolated groups (Ten animals each); group I was administered normal saline and act as a control group. Group II was received Tenofovir at a dose of $10 \mathrm{mg} / \mathrm{kg} /$ day for two months by stomach gavage. Group III was received Tenofovir at a dose of 10 $\mathrm{mg} / \mathrm{kg} /$ day and vitamin $\mathrm{E}$ also was given $100 \mathrm{mg} / \mathrm{kg} /$ day for two months. Group IV was received Tenofovir $10 \mathrm{mg} / \mathrm{kg} / \mathrm{day}$ for two months then withdrawal of Tenofovir for one month.

\section{Methods}

Renal tissue sampling: Twenty-four hours after the last treatment, rats were sacrificed by decapitation and kidneys were rapidly excised from each animal, divided equally and washed with $0.9 \% \mathrm{NaCl}$ solution and distilled water, then blotted using filter paper. One portion was fixed in $10 \%$ neutral buffered formalin for histopathological examination and the other part was kept freeze under $-20{ }^{\circ} \mathrm{C}$ for peroxidation assays. The sampling process was performed after two months except control group (group I) where all rats were sacrificed at day 10th. Renal samples were washed with a phosphate buffered saline (PBS) solution, $\mathrm{pH} 7.4$ including $0.16 \mathrm{mg}$ $/ \mathrm{ml}$ heparin to get rid of red blood cells and clots preceding its dissection into specimen.

\section{Electron microscopic examination} technique: Using a sharp knife, each kidney was cut in a sagittal plane into two halves and small cubes were taken and kept in $5 \%$ glutaraldehyde in sodium cacodylate buffer at $4^{\circ} \mathrm{c}$ for 24-48 hours. Samples were washed in sodium cacodylates buffer at $\mathrm{pH} 1.5$ for three changes 20 minutes each Samples were post fixed in $1 \%$ osmium tetroxide for hours. Samples were washed in four changes of sodium cacodylates buffer, 20 minutes each. Samples were dehydrated in ascending grades of alcohol as follows $30 \%$ for 30 minutes, $50 \%$ for 30 minutes, $70 \%$ over night in a refrigerator $90 \%$ for $30-60$ minutes and then in $100 \%$ twice 30 minutes each. Samples then were put in the following solutions: Propylene oxide for 30-60 minutes, propylene oxide placed in Epons-Araldite formulation (1:1) over night, pure Epon- Araldite formulations for 30-60 minutes. The tissues were embedded in a pure Epon-Araldite mixture for 24 hours (Ayache et al., 2010). Tissues blocks were polymerized in an oven at $35^{\circ} \mathrm{c}$ for 24 hours, at $45^{\circ} \mathrm{c}$ for 24 hours and at $60^{\circ} \mathrm{c}$ for 24 hours. Two types of section cutting were done:

a) Semi thin sectioning at 1umSpecimens were trimmed with a razor blade and stained with $2 \%$ aqueous toluidine blue then dried by absolute ethanol on a hot plate at $40^{\circ} \mathrm{c}$.

b) Ultrathin sectioning at $50 \mathrm{~nm}$ were recommended on cooper grids examined by a transmission Jeol=JEM=100CXII electron microscope and photographed at the electron microscope unit of Assiut university.

\section{Alkaline phosphatase reaction by Immuno-histochemical technique}

Paraffin sections were cut into $5 \mu \mathrm{m}$ thick slices. After paraffin was removed using xylene, the sections were incubated in $0.3 \%$ hydrogen peroxide in absolute methanol for $30 \mathrm{~min}$ at room temperature in order to inactivate endogenous peroxidase. The sections were then washed with $0.01 \mathrm{M}$ phosphate buffer, $\mathrm{pH} 7.2$ containing $0.85 \%$ sodium chloride (PBS) and 10\% normal horse serum was placed on the sections for 20 min at room temperature. After washing with PBS, anti-rat kidney ALP rabbit diluted 50-fold with PBS was applied to the sections and left for $30 \mathrm{~min}$ at room temperature (Chida, 1993). Thereafter, the sections were washed with PBS and incubated with an avidin-biotin-peroxidase complex (ABC) kit (Vectastain ABS kit; Vector Laboratories, Burlingame,CA).

\section{Morphometric \&Statistic analysis}


Estimation of the diameters of the glomeruli, proximal and distal convoluted tubules (maximum diameter) in the control and treated groups (at magnification of 400). The previous parameters were measured using Digimizer Image Analysis Program (MedCalc Software Co). Statistical analysis: Variables were represented by mean \pm SD (Mean \pm standard deviation of the mean).

\section{Results}

Table 1: Effect of Tenofovir $(10 \mathrm{ml} / \mathrm{kg})$, vitamin E $(100 \mathrm{mg} / \mathrm{kg})$, and their combination (Tenofovir + vitamin E) and withdrawal on glomerular, proximal and distal tubular diameter after two months of experiment were estimated from the paraffin sections analysis system (Leica Q500 MC). (Mean \pm SE) $(\mathrm{N}=10)$

\begin{tabular}{|l|l|c|c|c|}
\hline & \multicolumn{1}{|c|}{$\begin{array}{c}\text { Group } \\
\text { I }\end{array}$} & $\begin{array}{c}\text { Group } \\
\text { II }\end{array}$ & $\begin{array}{c}\text { Group } \\
\text { III }\end{array}$ & $\begin{array}{c}\text { Group } \\
\text { IV }\end{array}$ \\
\hline Glomeruli & $102.74 \pm 7.9$ & $79.57 \pm 8.8^{* * * *}$ & $100.68 \pm 2.8^{* * *}$ & $88.48 \pm 5.5^{* *}$ \\
\hline PCT & $44.51 \pm 1.8$ & $29.75 \pm 5.12^{* * *}$ & $41.14 \pm 2.44^{* * *}$ & $33.94 \pm 2.5 * *$ \\
\hline DCT & $33.33 \pm 3.12$ & $25.68 \pm 2.11^{* * *}$ & $32.37 \pm 2.05^{* * * *}$ & $29.44 \pm 1.01 * *$ \\
\hline
\end{tabular}

\section{Alkaline phosphatase reaction}

In control group positive reaction in the form of dark brown granules in the apical surface of proximal convoluted tubules (Fig. 3 a). In group II: negative Alkaline phosphatase reaction (Fig. 3 b). In group III: positive Alkaline phosphatase reaction (Fig.3 c). In group IV: Minimal positive Alkaline phosphatase reaction was observed in the form of minimal dark brown granules (Fig. $3 \mathrm{~d}$ ).

Morphometric results: Mean glomerular diameter in control group was $102.74 \pm 7.9$ while it was $79.57 \pm 8.8$ in group (II) which was marked decreased in comparison with control group while in group (III) it was $100.68 \pm 2.8$ which was nearby the diameter in control group. Glomerular diameter in group (IV) was $88.48 \pm 5.5$.

Mean PCT diameter in control group was $44.51 \pm 1.8$ while it was $29.75 \pm 5.12$ in group (II) which was marked decreased in comparison with control group while in group (III) it was $41.14 \pm 2.44$ which was nearby the diameter in control group. PCT diameter in group (IV) was 33.94 \pm 2.5 .

Mean DCT diameter in control group was $33.33 \pm 3.12$ while it was $25.68 \pm 2.11$ in group (II) which was marked decreased in comparison with control group while in group (III) it was $32.37 \pm 2.05$ which was nearby the diameter in control group, diameter in group (IV) was 29.44 \pm 1.01 . 

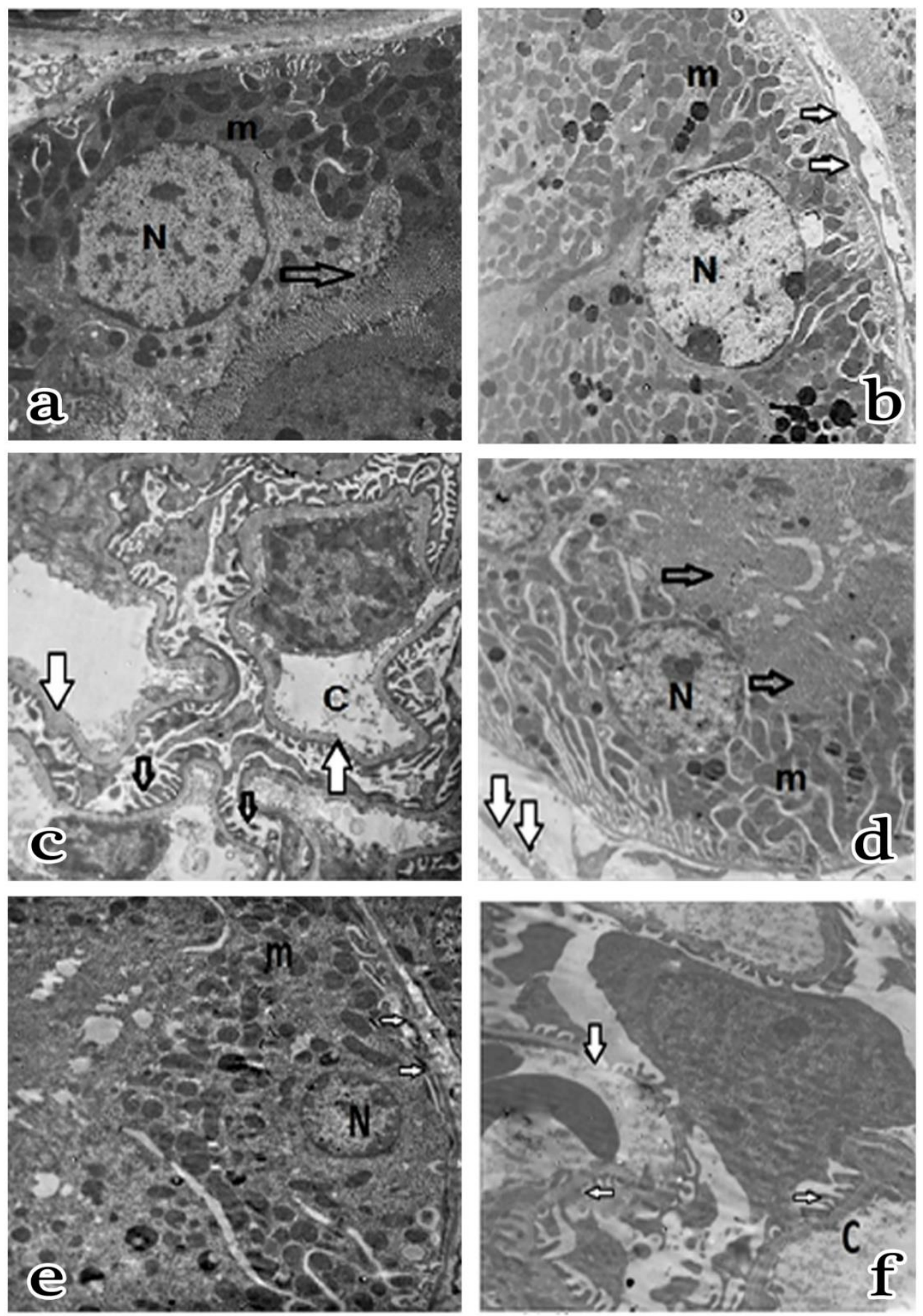

Fig. (1): Electro-photomicrographs of kidney sections from control and experimental group, represented, (Figs. a-c) normal control kidney sections in the proximal convoluted tubule from group I (Fig. a) showing normal rounded nucleus $(\mathrm{N})$ and multiple elongated basally arranged mitochondria $(\mathrm{m})$, regular apical microvilli (arrow). Distal convoluted tubule (fig. b) showing normal rounded nucleus (N) and multiple elongated basally arranged mitochondria $(\mathrm{m})$ and regular basement membrane (Thick arrow), glomerular capillaries (Fig. c) showing glomerular capillary (C) The capillary endothelium rest on basal lamina (arrow head). The podocytes have multiple pedicles (arrows). group II (Fig. d, e, f). proximal convoluted tubule (Fig. d) showing shrunken nucleus $(\mathrm{N})$, swollen mitochondria(m) with distortion of brush border(arrows), Distal convoluted tubule (Fig. e) showing shrunken nucleus (N), swollen mitochondria(m) with thickening of the basement membrane (Thick arrow). glomerular capillaries (Fig. f) showing irregular basal lamina in some areas (Thick arrow) and distorted pedicles (arrow). X5810 

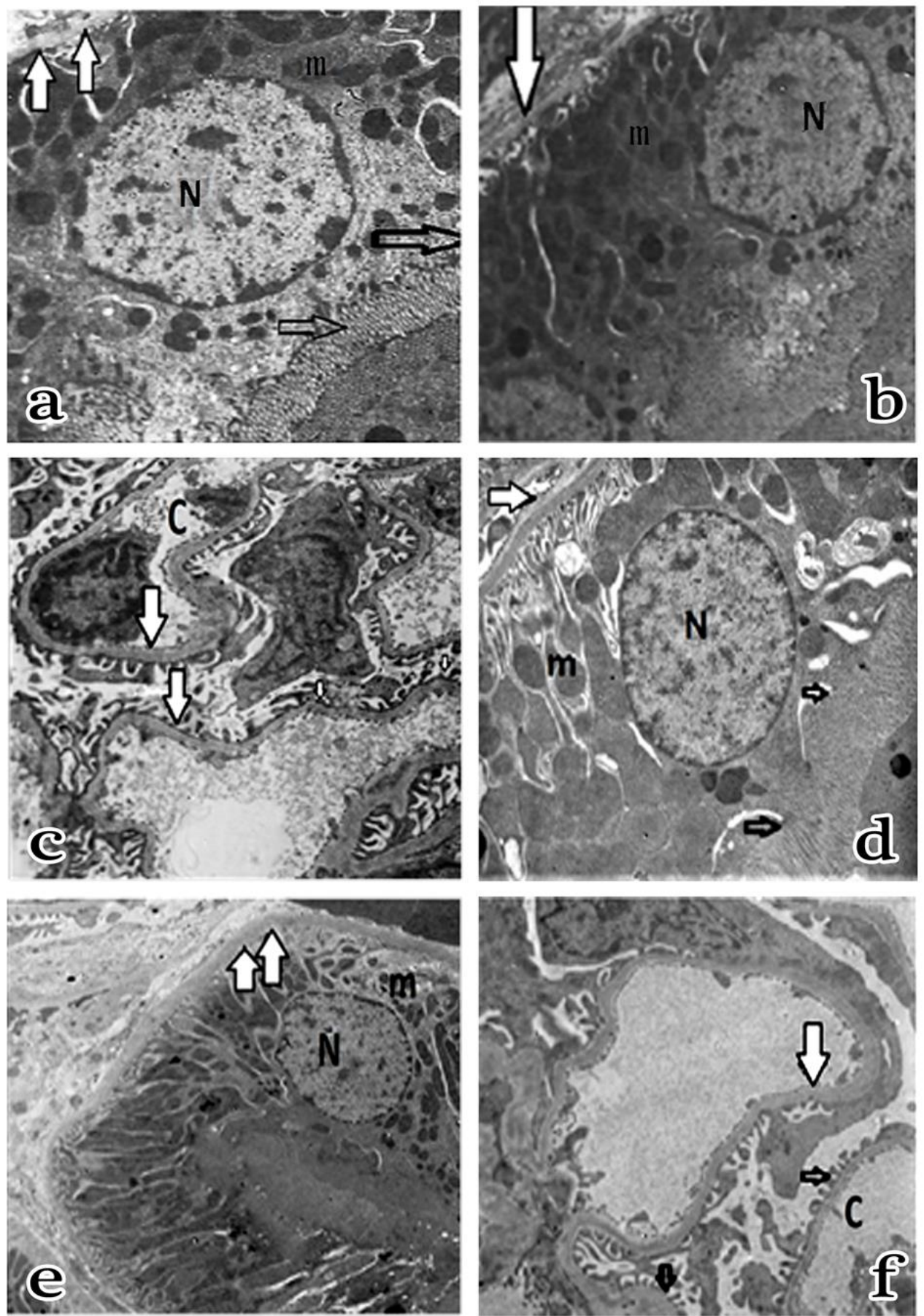

Fig. (2): Electro-photomicrograph of kidney sections from experimental groups, represented, (Figs. ac) represented group III. proximal convoluted tubule from group III (Fig. a) showing normal rounded nucleus $(\mathrm{N})$ and multiple elongated basally arranged mitochondria $(\mathrm{m})$, regular apical microvilli (arrow). Distal convoluted tubule (fig. b) showing normal rounded nucleus (N) and multiple elongated basally arranged mitochondria $(\mathrm{m})$ and regular basement membrane (Thick arrow), glomerular capillaries (Fig. c) showing glomerular capillary (C) The capillary endothelium rest on basal lamina (arrow head). The podocytes have multiple pedicles (arrows). group IV (Figs. d, e, f). proximal convoluted tubule (Fig. d) showing shrunken nucleus $(\mathrm{N})$, swollen mitochondria $(\mathrm{m})$ with distortion of brush border(arrows), Distal convoluted tubule (Fig. e) showing shrunken nucleus $(\mathrm{N})$, swollen mitochondria $(\mathrm{m})$ with thickening of the basement membrane (Thick arrow). glomerular capillaries (Fig. f) showing irregular basal lamina in some areas (Thick arrow) and distorted pedicles (arrow) X5810 

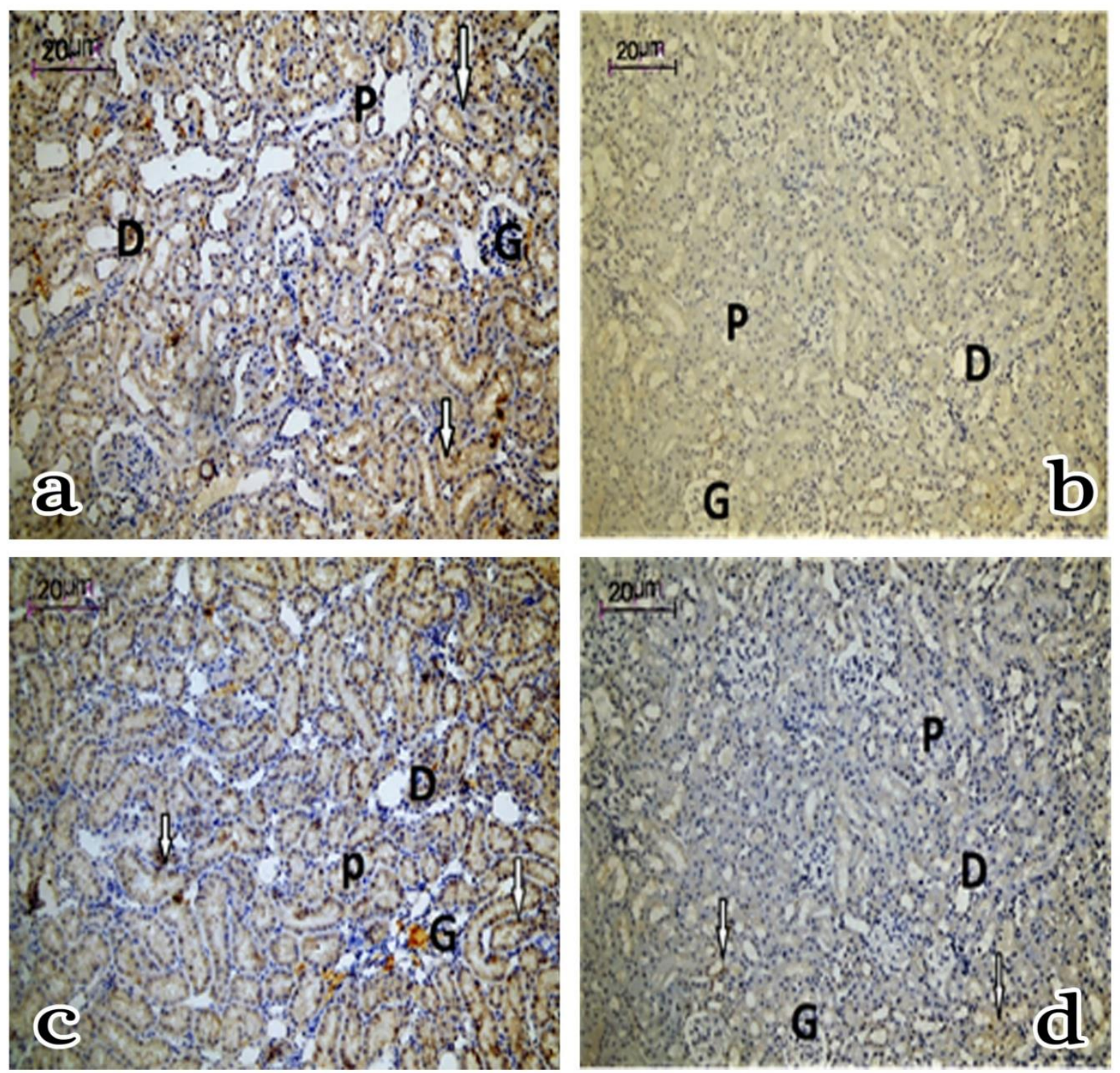

Fig. (3): Photomicrograph of kidney sections from control and experimental groups, represented Alkaline phosphatase reaction (Figs. a-d): normal control kidney sections (Fig. a) showing positive reaction in the form of dark brown granules in the apical part of proximal convoluted tubules (arrows). Group II (Fig. b) showing negative alkaline phosphatase reaction. Group III (Fig. c) showing positive reaction (arrows). Group IV (Fig. d) showing minimal positive reaction (arrows). Bars $=20 \mu \mathrm{m}$ in all parts.

\section{Discussion}

In the present work electron microscopic examination showing: In group II there was shrunken nucleus, mitochondria were swollen and irregular in shape. There was distortion of brush border, thickening of basement membrane as compared with the control group. Renal glomeruli showed thickening in the basal lamina with distorted pedicles. These results coincide with James et al. (2009) who reported that mitochondrial ultra-structural changes in the form of increased mitochondrial 
number, irregular shape were associated with the administration of TDF.

Alkaline phosphatase reaction was negative especially in the apical part of the proximal convoluted tubules when compared with control group. These results coincide with Jung et al. (2018) who reported several cases of renal tubular dysfunction and Fanconi syndrome in TDF-treated patients which lead to excessive urinary excretion of solutes handled by the proximal tubule such as phosphate, glucose, and bicarbonate.

As a result, hypophosphatemia was a possible complication in TDF treated patients. Pandit et al. (2012) explained that these changes were due to oxidative stress which caused loss of membrane fluidity, changes in membrane potential and an increase in membrane permeability. Another possible mechanism could be due direct kidney damage induced by TDF leading to dysfunction and disturbance in the biosynthesis evaluated biochemical parameters which lead to the elevation of serum concentrations of specific biochemical parameters; ALT, AST, ALP because of their release or secretions from the damaged tissues (Kuester et al., 2002).

In group III there was regular nucleus with regular nuclear membrane, Mitochondria were elongated basally located, PCTs showed normal brush border, glomerular capillaries showed regular basal lamina with regular multiple pedicles. These results coincide with Simona et al. (2015) who reported that vitamin E supplementation may be an effective accessory therapy to regulate superoxide generation in human mitochondrial ROS, monocytes and neutrophil, this protective effect on oxidative stress attenuates the onset and development of several chronic/degenerative diseases including CKD, Alkaline phosphatase reaction was positive when compared with group (II). These results were confirmed by Mine et al. (2009) reported that vitamin E supplementation increases alkaline phosphatase activity in the kidney.
In group IV there was some irregularity of nuclear membrane, swollen mitochondria with some distortion of brush border of PCTs, thickening of basement membrane at some areas, Glomerular capillaries showed thickening of basal lamina at some areas with pedicles distortion but less than group (II). These results were supported by Abhasnee et al. (2017) who detected improvement of urinary biomarkers after Tenofovir withdrawal which may represent an early sign of proximal tubular dysfunction recovery, Alkaline phosphatase reaction showed minimal positive reaction when compared with group (I) and (III).

In our study, morphometric results coincide with Post (2010) who reported that Tenofovir treated group had smaller renal corpuscle and glomerular diameter compared with control group. Also (Nel et al., 2017) explained that renal corpuscle diameter and renal space after TDF administration had smaller diameter compared with the control group suggesting that TDF decreased glomerular filtration rate possibly due to its effect on tubular function.

\section{Conclusion}

Tenofovir should be used with caution in patients with high risks of renal dysfunction and even a mild degree of renal dysfunction.

\section{Acknowledgments}

Great thanks to Professor Dr. Dorreia Abd Allah Mohamed Zaghloul for guidance, cogent commentary, experience of science and knowledge and effective and objective supervision. Great thanks to Assistant Prof. Eman Ahmed Abd El Rahim for her enthusiastic support, motivation, inspiration. A particular note of thanks is given to Dr. Salwa Mohamed Ouies for her valuable remarks, assistance and substantial advices.

\section{References}

Abhasnee S, Pawin N, Jirachaya W, Areepan S, and Bunyong P (2017). Reversal of Proximal Renal Tubular Dysfunction after Nucleotide 
Analogue Withdrawal in Chronic Hepatitis B, BioMed Research International pp:8.

Ayache J, Beaunier L, Boumend J and Ehret G (2010). Sample preparation handbook for transmission electron microscopy :423-482.

Bacha WJ and Bacha LM (2012). Color atlas Veterinary histology Lippincott Williams \& Wilkins 2nd ed. Ch. 14: 63.

Buti M and Homs M (2012). Tenofovir disoproxil fumarate in the treatment of chronic hepatitis. Expert Rev Gastroenterol Hepatol;6(4):413442.

Chida K (1993). Immunohistochemical detection of alkaline phosphatase in formalin fixed and paraffinembedded rat organs by means of avidin-biotin peroxidase complex method. Okajimas Folia Anat. Jpn, 70(5):203-208.

Choe, Eunok, Min and David B (2009). "Mechanisms of Antioxidants in the Oxidation of Foods". Comprehensive Reviews in Food Science and Food Safety. 8 (4): 345-358.

Cihlar T, Ho ES, Lin DC and Mulato AS (2001). Human renal organic anion transporter 1 (HOAT1) and its role in the nephrotoxicity of antiviral nucleotide analogs. Nucleosides and Nucleic Acids ;20(4-7):641-648.

Das UN, Podma M, Sogar PS, Ramesh G and Koratkar R (1990). Stimulation of free radical generation in human leukocytes by various agents including tumor necrosis factor is a calmodulin-dependent process. Biochem Biophys Res Community 67:1030-1036.

Findlay M, Isles C (2015). Structure and Function of the Kidney. Clinical Companion in Nephrology. Cham, Springer International Publishing: 39

James J, Seyed H, Amy Hoying-Brandt, Elgin G, David M, Rodney R, Dung
$\mathrm{T}$, C Michael $\mathrm{R}$ and William $\mathrm{L}$ (2009). Tenofovir renal toxicity targets mitochondria of renal proximal tubules.;89(5)513-519.

Jung, Woo Ji, Jang and Young MD (2018). Effect of tenofovir on renal function in patients of chronjc hHepatitis B Volume 97(7).

Kuester RK, Waalkes MP, Goering PL, Fisher PL, McCuskey RS and Sipes IG (2002). Differential hepatotoxicity induced by cadmium in Fischer 344 and Sprague- Dawley rats. Toxicol. Sci.,65:151-159.

Lyseng-Williamson KA, Reynolds NA and Plosker GL (2005). Tenofovir disoproxil fumarate: a review of its use in the management of HIV infection; 65:413-432.

Meydani M, Fielding RA and Fotouhi N (1996). Vitamin E. In wolinsky I and Driskell JA (eds), Sport Nutrition vitamins and Minerals. New York: CRC Press:119-131.

Mine E, Ebru B, Mesut A Ayşe S (2009). Effects of oral vitamin $\mathrm{E}$ and /or Selenium on alkaline phosphatase activity in the liver, kidneys and heart of prednisolone-injected rats 16(1):329.

Nel S, Strijdom H, Genis A, Everson F, Van Wijk R, Kotze SH (2017). Histomorphometric effects of antiretroviral therapy combined with a high calorie diet on aortic perivascular adipose tissue. 119: 555-562.

Oluwatosin AA, Olubusuyi MA and Ebenezer OF (2012). Effect of tenofovir, an antiretroviral drug, on hepatic and renal functional indices of wistar rats: protective role of vitamin E, journal of Basic and Clinical Physiology and Pharmacology; vol 23(2):69-75.

Pandit A, Sachdeva T and Bafna P (2012). Drug-Induced Hepatotoxicity: A Review, Journal of Applied Pharmaceutical Science; 02 (05): 233-243. 
Pham PA and Gallant JE (2006). "Tenofovir disoproxil fumarate for the treatment of HIV infection." Expert Opin Drug Metab Toxicol 2(3): 459-469.

Post F (2010). Randomized comparison of renal effects, efficacy and safety with once daily abacavir/lamivudine versus tenofovir/emtricitabine, administered with efavirenz in antiretroviral-naïve, HIV-infected adults: 48-weeks results from the ASSERT study. Journal of acquired immune deficiency syndromes.55(1) PP 47-57.
Simona G, Alessandra D, Paola T, Antonio $\mathrm{L}$ and Gianluigi $\mathrm{Z}$ (2015). Mitochondria: a new therapeutic target in chronic kidney disease ; 12 : 49.

Traber MG (2006). Vitamin E. In: Shils, M.; Shike, M. and Ross A.C. (eds). Modern Nutrition in Health and Disease. 10th ed. Baltimore: Lippincott Williams \& wilkins :396411.

Ustianowski, Andrew, Arends and Joop E (2015). Tenofovir: What We Have Learnt After 7.5 Million PersonYears of Use. Infectious Diseases and Therapy. 4 (2): 145-157. 